\title{
A Morphological View on Mitochondrial Protein Targeting
}

\author{
IDA J. VAN DER KLEI, MARTEN VEENHUIS, AND WALTER NEUPERT \\ Institute for Physiological Chemistry, University of Munich, 80336 Munich, Germany (I.J.v.d.K., W.N.), and Laboratory for Electron \\ Microscopy, University of Groningen, Biological Centre, 9751NN Haren, The Netherlands (M.V.)
}

\section{KEY WORDS Mitochondrion, Contact sites, Protein translocation, Ribosomes, Import inter- mediates, Receptor proteins}

\begin{abstract}
Mitochondrial protein targeting includes both intramitochondrial sorting of proteins encoded by the organellar genome and import and subsequent sorting of nuclear encoded precursor proteins. Only a few proteins are encoded by the mitochondrial genome and synthesized in the organellar matrix. These include predominantly inner membrane proteins that are perhaps co-translationally inserted into this membrane. Biochemical data suggest that insertion into the inner membrane may be confined to the inner boundary membrane. Ultrastructurally, however, a preferential association of ribosomes with either inner boundary or cristae membranes has not been established,

The majority of the mitochondrial proteins are nuclear encoded and synthesized as precursors in the cytosol. Electron microscopic studies revealed that import of precursor proteins is generally confined to sites where both mitochondrial envelope membranes are closely apposed. In line with these observations, biochemical studies indicated that precursor proteins destined for the inner membrane or matrix have to interact with the energized inner membrane to allow complete passage of the precursor through the outer membrane. As a consequence, the mitochondrial envelope membranes have to be in close proximity at protein import sites.

In isolated mitochondria distinct sites (designated as contact sites) exist where both envelope membranes are closely apposed and presumably stably associated. In situ, however, mitochondrial boundary membranes are in close proximity over large areas that cover almost the entire mitochondrial periphery. Consequently, the relative area of the mitochondrial surface, where both boundary membranes are in sufficient proximity for allowing protein translocation, is generally larger in situ compared to that in isolated organelles.

Immunocytochemical localization studies showed a rather random distribution of components of the mitochondrial protein translocation machinery over the entire mitochondrial surface and not confined to contact sites.

Based on these ultrastructral data and recent biochemical findings we propose that mitochondrial protein import sites are dynamic in nature and include relatively labile regions of close association of the boundary membranes. In vitro, however, mitochondrial protein import may preferentially take place at or near the presumably stable contact sites. 101994 Wiley-Liss, Inc.
\end{abstract}

\section{INTRODUCTION}

In eukaryotic cells various specific cellular functions are compartmentalized in distinct subcellular organelles (e.g., nuclei, mitochondria, vacuoles, endoplasmic reticulum, and microbodies). The main function of mitochondria is the production of ATP via oxidative phosphorylation and the synthesis of several important metabolites, which makes these organelles essential for the viability of the cells. Morphologically, mitochondria are characterized by two envelope membranes, the outer and inner membrane, which enclose two subcompartments: the matrix, surrounded by the inner membrane, and the intermembrane space located between both envelope membranes.

Mitochondria are not synthesized de novo but develop from pre-existing ones by growth and division. As a consequence various newly synthesized components (lipids, proteins) have to be incorporated into already present organelles. The majority of the mitochondrial proteins are encoded by nuclear genes, whereas only few are encoded by the mitochondrial genome and synthesized within the matrix. These include parts of the components of the respiratory chain, the ATP synthase and proteins required for the expression of mitochondrial genes. Nuclear encoded precursor proteins, synthesized in the cytosol, generally contain $\mathrm{N}$-terminal presequences which direct them to their target organelle. Upon reaching the mitochondrial surface they are imported and then are subsequently sorted to their correct mitochondrial subcompartment. Several mitochondrial protein sorting pathways have been elucidated; these vary from the relatively simple insertion

\footnotetext{
Received July 7, 1992; accepted in revised form March 3, 1993.

Address reprint requests to W. Neupert, Goethestraße 33, 80336 Munich, Germany.
} 

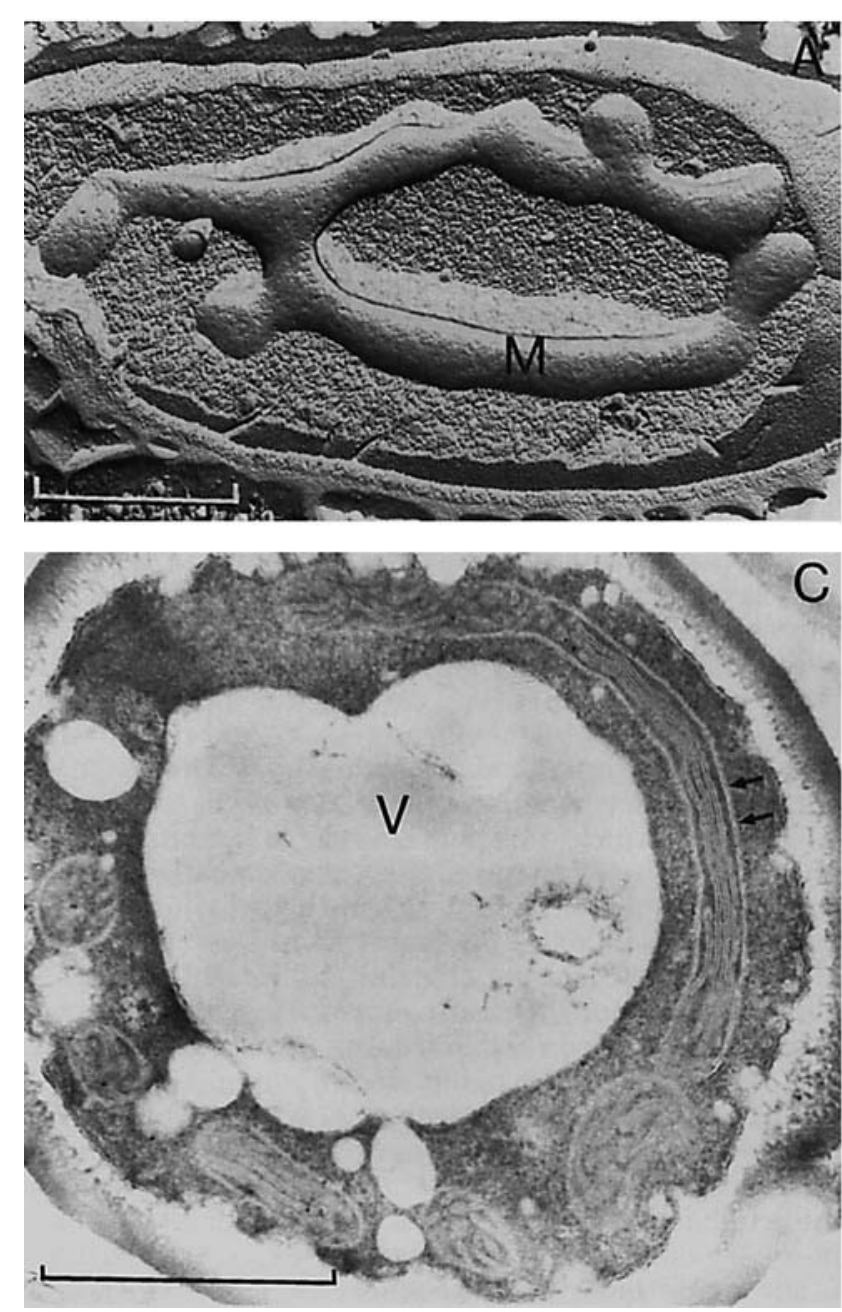

Fig. 1. Mitochondrial morphology in yeast. A: Freeze etch replica of a Candida utilis cell, showing the presence of a large, branched mitochondrion lying at the periphery of the cell (spray freezing, fracture plane through the mitochondrial outer membrane). B: Freeze etch replica showing the mitochondrial inner membrane and the sites of invagination into the cristae (arrows) (spray freezing using whole cells of Saccharomyces cerevisiae; the fracture plane is predominantly

mechanism of outer membrane proteins to very complex routes, in the course of which precursor proteins have to pass different membrane barriers in order to reach their functional location (Attardi and Schatz, 1988; Hartl et al., 1989; Hartl and Neupert, 1990; Pfanner and Neupert, 1990; Baker and Schatz, 1991). In this paper we summarize relevant morphological observations related to the sites of (intra)mitochondrial protein translocation. These include 1) the location of both mitochondrial and cytosolic ribosomes involved in the translation of mRNAs for mitochondrial proteins, 2) localization studies on precursor proteins en route to the mitochondrial matrix, and 3) localization studies on recently identified components of the mitochondrial protein import machinery.

Based on the morphological observations and closely
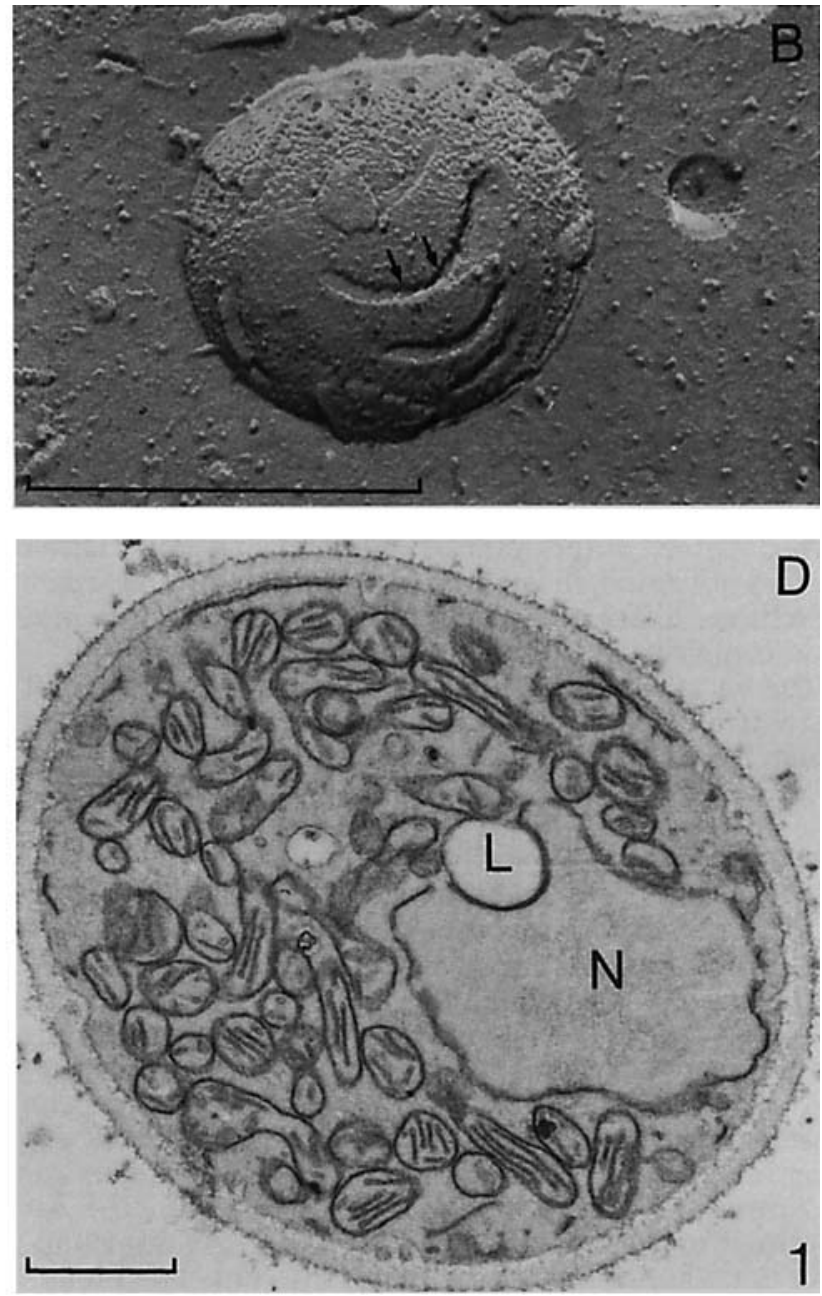

through the inner membrane). C: Thin section of a S. cerevisiae cell showing the elongated structure of mitochondria containing numerous cristae (arrows indicate the inner boundary membrane; glutaraldehyde fixed cell). D: Massive proliferation of mitochondria in the yeast Pichia pinus, grown in a medium containing ethanol as sole source of carbon and energy ( $\mathrm{KMnO}_{4}$ fixation). Bar represents $1 \mu \mathrm{m}$.

$\mathrm{L}$, lipid droplet; $M$, mitochondrion; N, nucleus; V, vacuole.

related biochemical findings we will present a model of mitochondrial protein translocation sites.

\section{MITOCHONDRIAL MORPHOLOGY}

The morphology of mitochondria may vary greatly, depending on the organism and cell type examined (Fig. 1). Its overall shape ranges from simply rounded organelles to elongated thread-like and/or branched structures. In addition, striking differences in the morphology of the inner membrane have been observed. This membrane can form variously shaped invaginations like folds/tubes into the matrix, which greatly increases its total surface area (Fig. 1B,C). This infolding part of the inner membrane, the so-called cristae membrane, is discriminated from the remaining area at the mitochondrial periphery, defined as the inner 
boundary membrane. Although these morphologically defined parts of the inner membrane are continuous in nature, they are not uniform in structure and function. Electron microscopic studies on negatively stained, broken inner membranes suggested that particles which reflect the ATP synthase were predominantly present at the cristae membranes instead of being randomly distributed over inner boundary and cristae membranes (Allmann et al., 1968). In addition, the surface of cristae and inner boundary membranes differ with respect to the density of anionic sites, which was visualized in electron microscopic studies using polycationic ferritin as probe (Hackenbrock and Miller, 1975). Further evidence that the inner membrane is not uniform in composition came from detailed biochemical and morphological studies on fractions obtained after mitochondrial subfractionation. These studies revealed that the distinct areas of the inner membrane differ with respect to both their protein and lipid composition (Werner and Neupert, 1972).

The variability in the mitochondrial shape, size, and ultrastructure is particularly evident in yeast cells, in which the mitochondrial proliferation often reflects specific environmental conditions (e.g., anaerobic/aerobic cultivation, fermentable/non-fermentable carbon sources) (see Fig. 1).

When examining the mitochondrial substructure in situ distinct differences are encountered related to the preparation methods used (e.g., whether cells are preserved by cryo- or chemical fixation) (Fig. 2A,B). In ultrathin sections, prepared from whole yeast cells after rapid freezing and subsequent freeze substitution, the outer and inner boundary membranes appear closely apposed (space between both membranes approximately $3-5 \mathrm{~nm}$; width across both membranes approximately $20 \mathrm{~nm}$ [Pfanner et al., 1992]) (Fig. 2A). An identical morphology has been reported for mitochondria in mammalian tissues, using the same techniques (Malhotra and Tewari, 1991). This close apposition results in a five-layered pattern, in contrast to the sevenlayered pattern, typical for two separated membranes as, for instance, observed for cristae membranes and the nuclear envelope. In chemically fixed cells, the distance between both boundary membranes is generally enhanced (total size across both membranes approximately $25-35 \mathrm{~nm}$ ) (Fig. 2B). As physical fixation is considered to better preserve the in vivo morphology compared to the routinely used chemical fixation techniques, a closer apposition of mitochondrial boundary may exist in vivo than is generally assumed.

More pronounced separation of the boundary membranes, paralleled by drastic alterations in the mitochondrial substructure, is only observed for isolated organelles. In the case of yeast mitochondria changes in the mitochondrial substructure already take place when intact protoplasts are slightly swollen, that is, in the initial step of the cell fractionation procedure, in that the boundary membranes separate. In addition, since mitochondria in fungi exist in an elongated, branched form, the isolation procedure undoubtedly causes rupture of the organelles, followed by resealing to form smaller, rounded structures (Fig. 2C).

The overall morphology of mitochondria inside intact protoplasts still largely reflects the in situ conformation, which is designated as "orthodox." However, more evident conformational changes occur as soon as protoplasts desintegrate (i.e., when the cell membrane is disrupted). In damaged protoplasts the matrix compartment of the mitochondria shrinks, paralleled by swelling of the intermembrane space (Fig. 2C). This so-called "condensed" conformation is generally observed for freshly isolated mitochondria.

In vitro the condensed conformation can be reversibly altered into the orthodox form by manipulating the metabolic state of the organelles. In addition, the conformation can be controlled by varying the osmolarity of the external medium (Hackenbrock, 1966). The latter phenomenon is related to the fact that the mitochondrial outer membrane contains large proteinaceous pores, whereas the inner membrane is permeable to water but not to ions or small molecules such as sucrose and hence osmotically active. In solutions with a high osmolarity (hypertonic) the matrix compartment shrinks (condenses), causing an increase in the volume of the intermembrane space paralleled by a swelling of the cristae and dissociation of the inner and outer boundary membranes. Under these conditions, however, distinct sites remain where boundary membranes are in close proximity, the so-called contact sites (Hackenbrock, 1968). When the osmolarity of the buffer is subsequently decreased to isotonic values, the morphology of the organelles largely reflects the orthodox conformation, a result of swelling of the matrix compartment. Upon incubation in hypotonic solutions, the matrix further expands accompanied by complete unfolding of the inner membrane, which provides further evidence that the inner boundary and cristae membranes are indeed continuous (Fig. 2D). In these mitochondria the outer membrane becomes disrupted: however, usually part of it remains associated with the inner membrane, most probably at sites which may reflect the contact sites observed in condensed organelles.

\section{CONTACT SITES}

Contact sites in condensed mitochondria isolated from Neurospora crassa have been studied in detail by electron microscopy (Rassow et al., 1989). Based on the analysis of serial sections of glutaraldehyde/osmium fixed, condensed organelles these sites were estimated to be $30 \mathrm{~nm}$ in diameter. The surface area of contact sites was calculated to occupy approximately $7 \%$ of the total surface of the outer membrane. The space between both membranes at contact sites was estimated to be $4-6 \mathrm{~nm}$, which is within the range observed for boundary membranes in freeze substituted yeast cells.

These ultrastructural data, which imply close association of two separate membranes at contact sites, contradict the semi-fusion model based on freeze-fracturing studies using isolated rat liver mitochondria (Venetië and Verkleij, 1982). The patchwork-like structure of the mitochondrial envelope, which is a result of fracture plane jumping between the boundary membranes, was proposed to occur at sites where the two apposing monolayers of each membrane were fused. However, using ultrathin sections of $N$. crassa mito- 

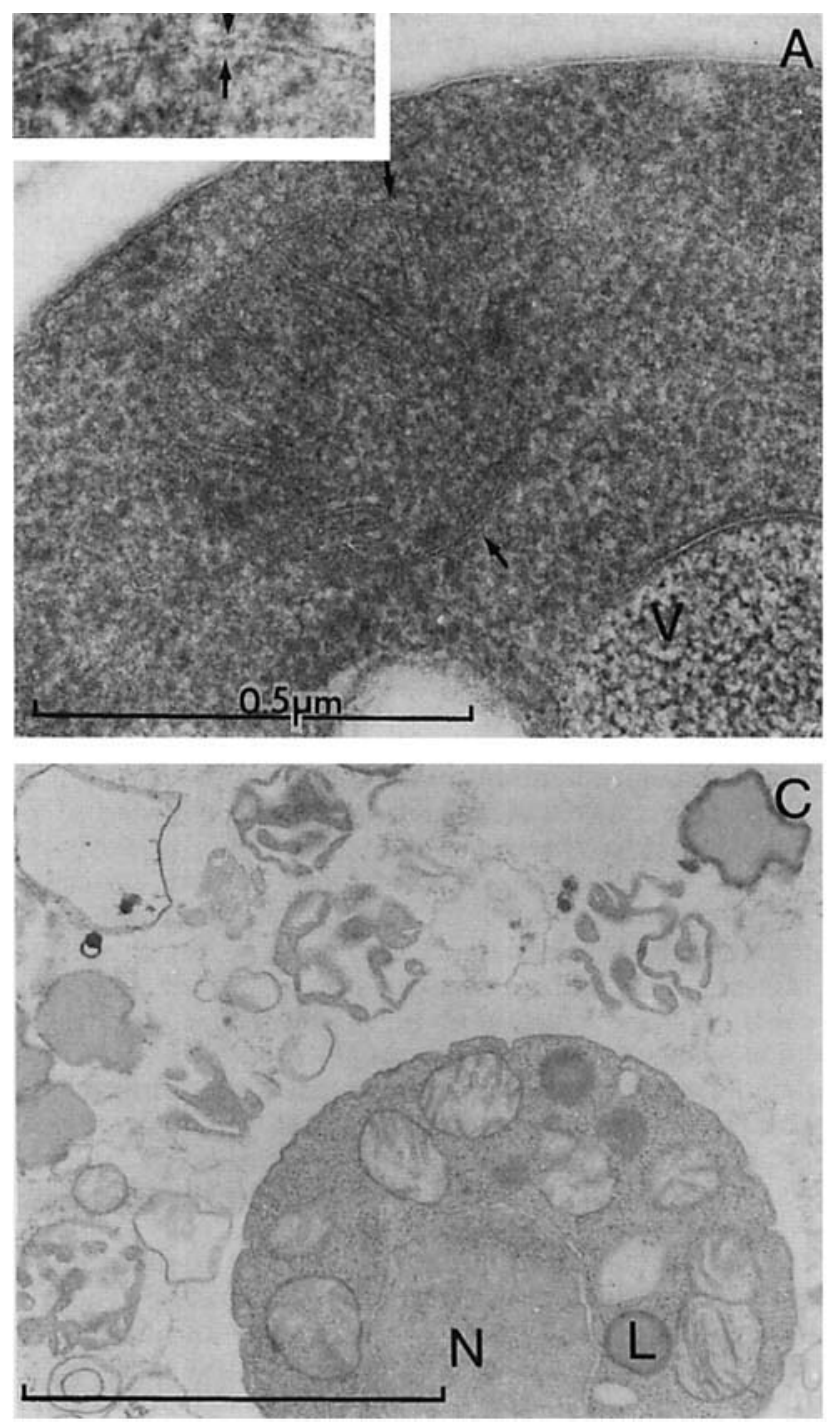

Fig. 2. Ultrastructural changes in mitochondrial substructure due to fixation and isolation procedures. A: Thin section of S. cerevisiae cell after rapid freezing and freeze substitution (uranylacetate, ethanol), showing a close apposition of the mitochondrial boundary membranes (arrows). A detail is shown in the inset. B: As A; however, cells were prefixed with glutaraldehyde. Note the separation of the boundary membranes compared to A. C: Morphology of mitachondria present inside intact protoplasts (orthodox) and released from broken

chondria (Rassow et al., 1989) obtained no morphological evidence that partial fusion of mitochondrial boundary membranes occurs at contact sites because the membranes were invariably found to be separated.

Different biochemical and ultrastructural studies indicated that contact sites are relatively stable structures. Firstly, upon preparation of mitoplasts (i.e., the inner membrane and matrix, essentially devoid of outer membrane and intermembrane proteins) part of the outer membrane remains tightly associated with the inner membrane. Secondly, contact sites remain intact after removal of most of the outer membrane by
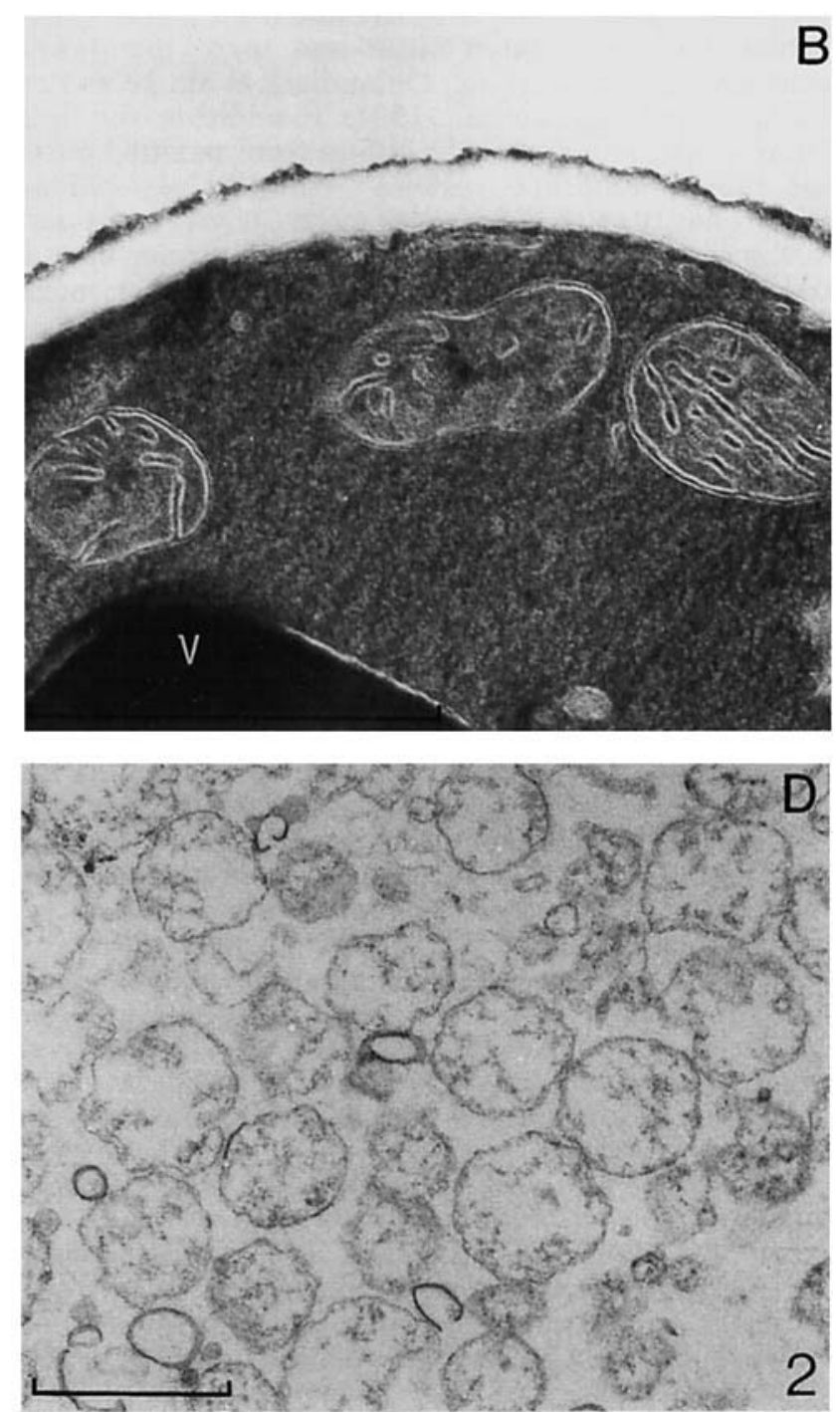

organelles (condensed). Yeast protoplasts obtained upon incubation with a lytic enzyme were incubated in a slightly hypotonic buffer which caused disruption of part of the protoplasts (glutaraldehyde fixation). D: Mitoplasts obtained upon incubation of isolated yeast mitochondria in a hypotonic buffer (glutaraldehyde fixation). Bar represents $1 \mu \mathrm{m}$ unless indicated otherwise. L, lipid droplet; $N$, nucleus; $\mathrm{V}$, vacuole.

mild detergent treatment. For instance, upon removal of the major part of the outer membrane by digitonin, remnants of these membranes formed inside-out vesicles which occasionally remained associated with the mitoplasts (Hackenbrock and Miller, 1975). Thirdly, contact sites are assumed to resist strong shearing forces. Upon sonication and subsequent separation of submitochondrial particles by sucrose gradient centrifugation, frequently a fraction of intermediate density between outer and inner membrane vesicles is found, which contains marker proteins of both membranes. Biochemical analyses revealed that, in these interme- 
diate density fractions (IDF) isolated from organelles of various sources, certain outer and inner membrane proteins are enriched (e.g., Ohlendieck et al., 1986; Pon et al., 1989; Lithgow et al., 1991). In addition, the lipid composition quantitatively differs from purified outer and inner membrane vesicles (Ardail et al., 1990a). These quantitative differences might, however, in part be the consequence of enrichment of the inner boundary membrane in the IDF, whereas the inner membrane fractions mainly contain cristae membranes.

Electron microscopic data reported so far revealed no common substructure of the membranes present in IDFs; however, the variations observed might reflect differences in the sources of the organelles and in the preparation procedures. In the case of rat liver mitochondria (upon sonication) right-side-out outer membrane vesicles, enclosing right-side-out inner membrane vesicles, were observed in freeze etch replicas (Ohlendieck et al., 1986). In contrast, the IDF obtained from osmotically lysed mouse liver mitochondria (without sonication) contained inner or outer membrane sheets attached to outer or inner membrane vesicles, respectively (freeze-fracturing studies [Ardail et al., $1990 \mathrm{~b}])$. When isolated from sonicated yeast mitochondria the IDF contained predominantly (leaky) insideout outer membrane vesicles, associated to right-sideout inner membrane vesicles (thin sections of cytochemically stained fractions) (Pon et al., 1989).

Although the above observations imply that contact sites are relatively stable, a more dynamic nature of contact sites has been proposed as well. This may in part be due to circumventional use of the term mitochondrial contact site as so far no general consensus for this term exists.

A dynamic nature of contact sites was proposed based on criteria that differ from the above stated ones, namely fracture plane deflections between both boundary membranes in freeze etch replicas (Knoll and Brdiczka, 1983) and sites where creatine kinase, an intermembrane space enzyme, displays enzyme activity (cytochemical staining) (Biermans et al., 1989, 1990). However, firstly, the specificity of both criteria to demonstrate close apposition of mitochondrial boundary membranes is still a matter of debate, whereas these sites may include rather labile areas of close apposition rather than reflect exclusively the contact sites encountered in, for instance, condensed isolated organelles.

To prevent any misinterpretation we use the term contact site in this review solely for those ultrastructurally detectable sites in condensed isolated organelles where both boundary membranes are in close proximity and the sites where the outer membrane remains attached to mitoplasts. Biochemically, contact sites are more difficult to define, but assumed to be enriched in fractions of intermediate density obtained upon mitochondrial subfractionation. Several models have been proposed for the nature of contact sites, which generally share the view that these structures are due to interactions between peripheral membrane/ intermembrane space proteins-for example, association of the outer membrane located porin with the inner membrane protein ADP/ATP carrier either directly
(McEnery et al., 1992) or indirectly via intermembrane space located kinases (Biermans et al., 1990; Brdiczka et al., 1990; Rojo et al., 1991; for review see Brdiczka, 1991). However, also in these cases the proposed proteins may be involved in labile associations of the mitochondrial envelope membranes, rather than represent contact sites. Moreover, conclusive evidence is lacking to designate any component to be responsible for the formation/maintenance of either labile mitochondrial membrane associations or contact sites.

The physiological significance of the closely apposed membrane configuration is most probably that it facilitates various exchange reactions between both envelope membranes, including energy, lipid, and protein transfer (Nicolay et al., 1990; Ardail et al., 1991; Brdiczka, 1991; McEnery et al., 1992; Wienhues and Neupert, 1992).

\section{SORTING OF MITOCHONDRIALLY SYNTHESIZED PROTEINS}

Only a small number of mitochondrial proteins are encoded by the mitochondrial genome. These proteins are synthesized in the matrix by a system which displays remarkable similarities with the bacterial protein synthesis apparatus, which may be interpreted to be the result of the evolutionary origin of the organelles from prokaryotic endosymbiotic ancestors. Mitochondrial ribosomes have generally lower sedimentation constants compared to their cytosolic counterparts. Vignais et al. (1969) reported the presence of aligned polysomes in isolated yeast mitochondria (Candida utilis), which were predominantly attached to the inner membrane both at the cristae and boundary membrane. A similar mitochondrial ribosome distribution was observed in thin sections of whole Saccharomyces cerevisiae cells (Watson, 1972).

Biochemical studies on mitochondrial ribosomes led to the conclusion that translating mitochondrial ribosomes are predominantly membrane-bound (Marzuki and Hibbs, 1986). The rationale behind this may be that these ribosomes mainly synthesize hydrophobic inner membrane proteins, which are co-translationally inserted into the inner membrane.

If this assumption is correct, translating ribosomes must be located at the sites of protein insertion. Based on the electron microscopic observations mentioned above protein insertion may not be confined to either cristae or inner boundary membranes, but occur randomly over the inner membrane. In contrast, biochemical studies, in which the incorporation of radiolabeled amino acids into matrix synthesized inner membrane proteins was followed, did suggest that newly synthesized proteins first insert into the inner boundary membrane, possibly at a position where the inner membrane merges into cristae membranes, and subsequently laterally migrate into the cristae (Werner and Neupert, 1972). According to this model mitochondrial ribosomes are expected to be enriched at the inner boundary membrane. To elucidate the reasons behind this discrepancy more extensive ribosomal location studies have to be carried out. However, conclusive knowledge on the distribution of the inner membrane protein insertion sites awaits the identification and 
(immunocytochemical) localization of components of the inner membrane protein insertion machinery.

\section{MITOCHONDRIAL PROTEIN IMPORT SITES}

Nuclear-encoded proteins have been described to constitute the majority of components of all mitochondrial subcompartments (Hartl et al., 1989). Most of these components are synthesized as precursors that must cross the outer membrane and interact with the inner membrane before reaching their functional locations (excluding outer membrane proteins and two intermembrane space proteins which follow an exceptional pathway: cytochrome c (Stuart et al., 1990) and cytochrome c heme lyase [Lill et al., 1992]).

The establishment of an in vitro protein import assay for mitochondria enabled extensive biochemical analysis of mitochondrial protein translocation, which resulted in the dissection of the import pathway in several distinct stages (Hartl et al., 1989). In addition, it is possible to manipulate precursor proteins to accumulate at different intermediate stages of translocation. Recent in vitro studies have indicated that precursor proteins en route to the inner membrane can be accumulated at a stage where they are largely exposed to the intermembrane space but still bound to either the outer or inner membrane. However, intermediates which are freely soluble in the intermembrane space were never observed (Rassow and Pfanner, 1991). Completion of translocation through the outer membrane may require interaction of the outer membrane associated precursor with the inner membrane. As a consequence, these precursor proteins may only reach the inner membrane at sites where both mitochondrial envelope membranes are in close proximity.

\section{SITES OF RIBOSOME BINDING AT THE MITOCHONDRIAL SURFACE}

The first indication that mitochondrial protein import occurs predominantly at sites where both boundary membranes are closely apposed came from electron microscopic studies by Kellems et al. (1975). In ultrathin sections of glutaraldehyde/osmium-fixed yeast spheroplasts, cytoplasmic ribosomes were found to be attached to the mitochondrial outer membrane. Detailed biochemical analyses revealed that these membrane-bound ribosomes translated predominantly mRNAs for mitochondrial precursor proteins (Ades and Butow, 1980; Suissa and Schatz, 1982). Since association of ribosomes was significantly reduced in starving cells, binding was considered to be mediated by nascent precursor proteins at mitochondrial protein import sites. Ribosomes attached in vivo remained associated with the mitochondrial outer membrane when the organelles were isolated in the presence of $1 \mathrm{mM} \mathrm{Mg}^{2+}$ (Kellems et al., 1975). The association was particularly evident when mitochondria were isolated from cycloheximide (CHI) treated spheroplasts. CHI inhibits cytosolic protein synthesis, leaving the nascent polypeptide chain attached to the ribosome. In isolated mitochondria with an orthodox configuration, the bound ribosomes covered nearly the entire mitochondrial periphery. However, in condensed mitochondria it became evident that ribosome binding was limited to areas where both boundary membranes were in close proximity, which might reflect the contact sites. However, it cannot be excluded that (part of) these sites are instead a result of (stuck) nascent polypeptide chains that were inserted into the inner membrane or already reached the matrix, while the ribosome-bound C-terminal part was still at the surface of the organelle.

To summarize, the above findings suggest that binding of ribosomes to the mitochondrial surface in $\mathrm{CHI}$ treated cells occurred at protein import sites, mediated by bound, nascent polypeptides, and that these sites are largely confined to those regions where both boundary membranes are in close proximity. This location of protein translocation sites is in agreement with our current view, based on later biochemical studies, that most precursor proteins have to interact with the inner membrane to allow complete passage through the outer membrane. An intriguing question remains, however, namely how mitochondrial protein import sites are formed and what their distribution is over the mitochondrial surface. Do they, for instance, reflect the relatively stable contact sites, which are encountered in condensed isolated organelles, or can they reversibly be formed over the entire mitochondrial periphery, wherever both membranes are closely apposed?

\section{IMPORT INTERMEDIATES GENERATED IN VITRO}

Another important finding with in vitro import experiments has been that stable two-membrane spanning import intermediates can be generated. Such intermediates, for instance, accumulate when the rate of in vitro protein import is slowed down by lowering incubation temperatures (Rassow et al., 1989) or when (artificial) precursor proteins are used which carry a tightly folded domain. As mitochondrial precursor proteins have to unfold during translocation across the envelope membranes, these tightly folded domains prevent completion of import (Schleyer and Neupert, 1985; Eilers and Schatz, 1986; Schwaiger et al., 1987; Vestweber and Schatz, 1988; Rassow et al., 1989).

The relatively stable translocation intermediates allowed for the first time a direct visualization of a known precursor protein en route to the mitochondrial matrix (Schwaiger et al., 1987). The precursor protein of a subunit of the ATPase (the $\beta$ subunit of the $F_{1}$ ATPase, which is associated with the inner membrane exposed to the matrix) was bound to antibodies raised against the C-terminal part of the protein, thereby introducing a stable folded domain. In vitro, upon incubation with isolated $N$. crassa mitochondria, the protein became partly imported into the matrix (based on $\mathrm{N}$-terminal processing by the matrix peptidase). The C-terminal part, however, was still accessible to externally added proteases, indicating that this part was not completely translocated into the organelle (Schleyer et al., 1985). Mitochondria containing the two-membrane spanning translocation intermediates were incubated with protein A/gold to label the prebound antibodies and subsequently fixed and embedded for electron microscopy. In thin sections significant enrichment of the labeling was observed at sites were outer and inner membranes were closely apposed, which in fact is the 
only possible location for a two-membrane spanning intermediate and therefore confirmed the biochemical data. Subsequent experiments suggested that these sites reflect contact sites as labeling was still evident in mitoplasts prepared from the labelled organelles at those sites where remnants of the outer membrane remained attached to the inner membrane (Schwaiger et al., 1987). Moreover, upon sonication and subsequent separation of submitochondrial particles by sucrose gradient centrifugation, the import-intermediate cofractionated with particles of intermediate density (IDF; see above). Similar results were obtained by Pon et al. (1989) using an artificial precursor protein, which was stalled during import into the matrix due to the presence of a stably folded protein, covalently coupled to its C-terminus. Electron microscopic observations revealed that the IDF of yeast organelles, containing the import intermediates, consisted of right-side-out inner membrane vesicles attached to leaky outer membrane vesicles (Pon et al., 1989). Artificial precursor proteins containing tightly folded domains have also been used to estimate the distance across both membranes which is spanned by mitochondrial precursors. For this purpose fusion proteins were constructed consisting of a mitochondrial presequence fused to dihydrofolate reductase (DHFR), a protein which remains stably folded upon incubation with the substrate analogue methotrexate (MTX). Several hybrid proteins consisting of portions of various lengths of cytochrome $b_{2}$ fused to the entire DHFR were incubated with isolated mitochondria in the presence of MTX. Proteolytic cleavage by the matrix located processing peptidase only occurred with those fusion proteins which contained a portion of at least 82 amino acids of cytochrome $b_{2}$ (Rassow et al., 1990). Because 31 amino acids were removed by the peptidase, about 50 amino acid residues were sufficient to span both boundary membranes. The length of 50 amino acids in a fully extended conformation corresponds to approximately $18 \mathrm{~nm}$, which is similar to the thickness of contact sites assessed by electron microscopy.

The above experiments imply that accumulation of translocation intermediates in vitro occurs at sites where the distance across both boundary membranes is up to $18 \mathrm{~nm}$ long (Rassow et al., 1990). These sites may reflect contact sites because they remain intact upon preparation of mitoplasts (Schwaiger et al., 1987) and co-fractionate with contact sites in intermediate density fractions (Schwaiger et al., 1987; Pon et al., 1989), indicating a relatively stable nature. However, it cannot be excluded that under the above circumstances the association of the membranes is (partly) mediated by stuck import intermediates.

\section{COMPONENTS OF THE MITOCHONDRIAL PROTEIN IMPORT MACHINERY}

Recently, several components of the mitochondrial import machinery have been identified. Although various distinct mitochondrial protein translocation pathways exist, the import machinery involved in translocation of matrix proteins plays a more general role in that it also is involved in import of the bulk of precursor proteins destined for other mitochondrial subcom- partments. The latter precursor proteins, however, either branch off at an early stage or require additional steps to reach their final destination. Upon synthesis in the cytosol, precursors of matrix proteins bind to proteinaceous receptors at the surface of the outer membrane. Next, they insert in this membrane at a general insertion site (designated GIP), which consists of several proteinaceous components (Kiebler et al., 1990; Söllner et al., 1992). Subsequently, the inserted polypeptide chain interacts with the inner boundary membrane, a process which is dependent on a membrane potential, negative inside, which possibly drives import of the positively charged presequence into the matrix by an electrophoretic effect (Martin et al., 1991). Finally, import into the matrix is completed in an ATPdependent fashion. The various components involved in the above translocation processes and those which mediate subsequent modification and assembly steps were recently reviewed in detail by several authors (Baker and Schatz, 1991; Pfanner et al., 1991; Wienhues and Neupert, 1992). Here, we will discuss only those proteinaceous components which have been localized so far by ultrastructural methods.

\section{IMMUNOCYTOCHEMICAL LOCALIZATION OF RECEPTOR PROTEINS}

The initial recognition that proteinaceous components, exposed on the mitochondrial surface, are involved in recognition of cytosolic precursor proteins came from the finding that pretreatment of isolated organelles with proteases abolished import. So far, three outer membrane proteins have been reported to act as (or being part of) receptors. Firstly, a mitochondrial outer membrane protein with an apparent molecular weight of $19 \mathrm{kD}$ (MOM19) was shown to be involved in the import of many different precursor proteins into $N$. crassa mitochondria, including precursor proteins destined for the outer membrane, the inner membrane, the intermembrane space, and the matrix (Söllner et al., 1989). In the same organism MOM72 was identified as another mitochondrial receptor, mainly involved in the recognition of the ADP/ATP carrier, located in the inner membrane (Söllner et al., 1990). A homologous protein, MAS70, isolated from yeast mitochondria, was shown to be involved in mitochondrial protein translocation as well, in that it accelerates import of several, but not all, precursor proteins (Hines et al., 1990).

Immunolabeling of ultrathin cryosections of isolated organelles revealed that MOM19 was located at the outer membrane. When isolated organelles were incubated with antibodies and protein A/gold prior to fixation and embedding, labeling at the outer membrane was also evident, suggesting exposure of MOM19 protein at the outer surface of the organelles (Fig. 3). Quantification of the labeling patterns revealed no significant enrichment of MOM19 at any site of the membrane.

Similar studies were carried out using antibodies raised against MOM72. As with MOM19, MOM72 also appeared to be exposed to the outer surface but, in contrast to MOM19, was approximately fivefold enriched in contact sites, compared to the remainder of the outer 


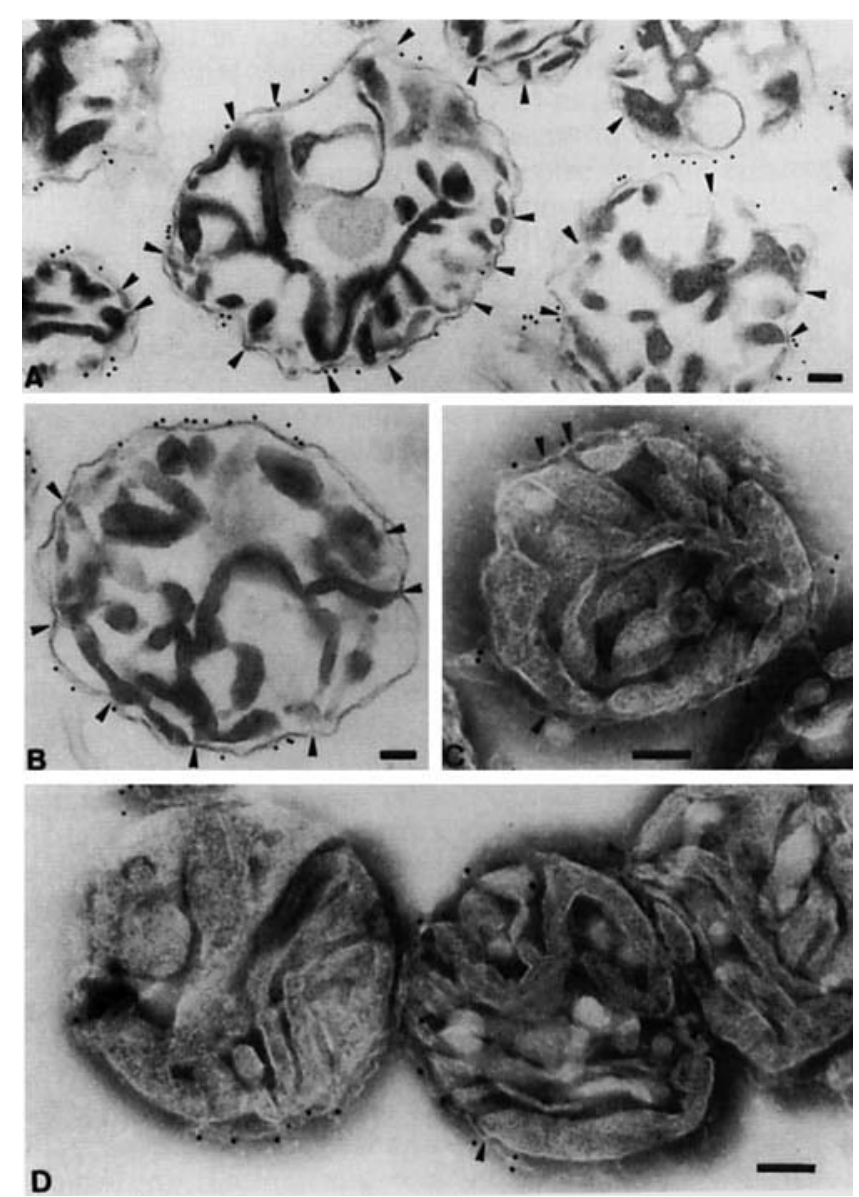

Fig. 3. Immunocytochemical localization of MOM19 in mitochondria isolated from $N$. crassa. A,B: Ultrathin sections of plastic embedded organelles, which were prelabelled with anti-MOM19 and decorated by protein A/gold. Gold particles are on the periphery of the outer membrane (A,B). C,D: Cryosections are shown, which were labelled with MOM19 antibodies and protein A/gold. The gold particles are predominantly on or close to the outer membrane. Contact sites are indicated by arrowheads. Bar represents $0.1 \mu \mathrm{m}$. (Reproduced from Söllner et al., 1989, with permission of Cell Press.)

membrane (based on quantitation of gold particles using isolated condensed organelles). The homologous protein in yeast, MAS70, showed a similar distribution pattern.

Using anti-idiotype antibodies, raised against a chemically synthesized signal peptide, Pain et al. (1990) identified an integral mitochondrial membrane protein (p32), which was assumed to act as a receptor protein in yeast; this was based on the finding that $\mathrm{F}_{\mathrm{ab}}$ fragments inhibited in vitro import of precursor proteins and the antibodies could specifically immunoprecipitate a precursor-p32 complex. Pre-embedding immunolabeling experiments using both the anti-idiotype- and an anti-p32 antibody showed that the antigenic sites were exclusively present on the outer membrane at sites where both membranes were closely apposed. Whether these sites represent contact sites is not clear because the organelles used were not in a condensed conformation.
Sequence comparisons revealed that p32 is identical to a protein identified as the inner-membrane located phosphate carrier (Murakami et al., 1990; Zara et al., 1991). Although it is difficult to reconcile the latter with the presumed location of p32, some possible explanations will be discussed.

Firstly, the antibodies used may have cross-reacted with an outer membrane protein involved in protein translocation. An appropriate candidate may include MOM38 (see below), which contains a stretch of approximately 70 amino acid residues, which is similar to the phosphate carrier (Kiebler et al., 1990; Zara et al., 1991). However, this is not a very likely explanation for the observed labeling pattern because MOM38 is randomly distributed over the outer membrane (Kiebler et al., 1990). Secondly, the reported contact site location might be an artefact owing to the immunolabeling techniques used. In principle, application of preembedding immunolabeling on intact mitochondria selectively leaves the outer surface of the organelle as putative location for accumulation of gold grains. Antigenic sites inside the organelles can only be labelled when the membranes are sufficiently permeable for both (primary and secondary) antibodies and gold particles.

In order to avoid possible misinterpretations, immunolabeling of ultrathin sections (i.e., post-embedding labeling) is therefore indispensible for confirming protein locations determined by pre-embedding labeling techniques.

\section{IMMUNOCYTOCHEMICAL LOCALIZATION OF A COMPONEN'T OF THE GENERAL PROTEIN INSERTION SITE}

Apart from the above mentioned receptor proteins, components have been identified which are assumed to be part of the protein translocation machinery. ISP42 was identified in yeast organelles by crosslinking to a membrane spanning import intermediate (Vestweber et al., 1989). MOM38, the $N$. crassa homologue of ISP42, is part of the import complex as suggested by the results from co-immunoprecipitation experiments with MOM19 and MOM72 (Kiebler et al., 1990). Immunolabeling of cryosections of $N$. crassa have revealed that MOM38 is randomly distributed over the outer membrane (Kiebler et al., 1990).

The above immunocytochemical localizations imply that receptor proteins and the protein import machinery are not exclusively located at contact sites, but instead randomly distributed over the outer membrane or in case of MOM72 only partially enriched at contact sites.

\section{CONCLUDING REMARKS AND PERSPECTIVES}

The visualization of precursor proteins en route to the mitochondrial matrix by ultrastructural techniques indicated that mitochondrial protein import occurs at sites where both boundary membranes are closely apposed. Unravelling of the consecutive steps in mitochondrial protein import processes by means of in vitro experiments offered an explanation for this finding. By arresting precursor proteins at different inter- 
mediate stages of import, it was found that precursor proteins destined for the inner membrane or matrix are never freely soluble in the intermembrane space, although membrane bound intermediates exposed to the intermembrane space can be accumulated under certain conditions (Rassow and Pfanner, 1991). As a consequence, release of these precursor proteins from the outer membrane may only occur upon interaction of the precursor with the inner membrane. Therefore, to allow translocation the distance between both boundary membranes must be sufficiently short to enable the precursor to interact with both envelope membranes at the same time.

In isolated mitochondria this structural prerequisite is predominantly fulfilled at contact sites. As a consequence, in vitro a preference may exist for protein import sites at or near these sites. This may especially be of importance when the organelles are in a condensed configuration during the import assays (hypertonic solutions or phosphorylating organelles) (Hackenbrock, 1966).

In contrast to the in vitro situation, in vivo a close apposition of the boundary membranes may exist over a much larger area covering almost the whole mitochondrial periphery (Pfanner et al., 1992) (Fig. 1A). Since, in addition, components of the mitochondrial protein translocation machinery are randomly distributed over the entire mitochondrial surface, we propose that in vivo mitochondrial protein import sites may occur all over the mitochondrial boundary except for those sites where the inner membrane dissociates from the outer membrane into cristae.

The absence of large areas of close apposition in isolated organelles implies that the association of the boundary membranes between contact sites is relatively labile. This is also suggested from the observations that chemical fixation of whole yeast cells and slight osmotic swelling of protoplasts causes separation of the boundary membranes. These observations may be related to our current view that the mitochondrial protein import machinery is dynamic in nature rather than being a fixed, stable import channel. This view is based on recent biochemical findings. Firstly, cytochrome c heme lyase (CCHL), which follows an exceptional pathway to the intermembrane space, uses the MOM19-GIP complex to translocate across the outer membrane, but in contrast to other proteins this translocation step is independent on an energized inner membrane. This finding suggests that the import machinery of the outer membrane can act independently from the insertion/import machinery in the inner membrane (Lill et al., 1992). Similarly, the inner membrane machinery can act independently of the outer membrane machinery, because precursor proteins can be imported into mitoplasts, directly through the inner membrane (Hwang et al., 1989). When the import machineries of both boundary membranes do indeed act either in parallel (e.g., in case of all inner membrane and matrix proteins) or independently (e.g., CCHL and mitoplast import), this excludes a stable, irreversible interaction of both import machineries at protein translocation sites (Glick et al., 1991; Pfanner et al., 1992). In addition, these findings give further evidence that protein import is not confined to contact sites, although these sites may be the "main entrance" for most precursor proteins in vitro.

Despite the progress in the field of mitochondrial biogenesis and function, a challenging problem has been paid little attention to so far, namely what contact sites actually are. Apart from the extensive ultrastructural studies on isolated condensed $N$. crassa mitochondria by Rassow et al. (1989), little information is available. Intriguing questions are, for instance, which components are responsible for the formation of contact sites and what the function of contact sites is. A possible role includes that the structures which form contact sites in vitro are involved in vivo in keeping the boundary membranes in sufficient proximity or at a defined distance. Furthermore, contact site components could be involved in the formation of cristae. Morphological studies on isolated organelles which are not completely condensed suggest that contact sites are present at those sites where the inner membrane merges into the cristae. Moreover, at these sites contact site components could have a barrier function and could be important for maintaining differences in composition of the cristae and inner boundary membranes. Consequently, it is interesting to know where components of contact sites are located in situ, as so far contact sites are only recognizable in condensed isolated organelles and mitoplasts.

Although our current view on mitochondrial protein import includes no direct, major function of contact sites on protein translocation, these may indirectly be important to fulfill ultrastructural prerequisites for protein import as indicated above.

Detailed morphological and biochemical analysis of contact sites may therefore supply important information for various lines of investigations in mitochondrial biogenesis and function and will be a challenging topic for future investigations.

\section{REFERENCES}

Ades, I.Z., and Butow, R.A. (1980) The products of mitochondriabound cytoplasmic polysomes in yeast. J. Biol. Chem., 255:99189924.

Allmann, D.W., Bachmann, E., Orme-Johnson, N., Tan, W.C., and Green, D.E. (1968) Membrane systems of mitochondria. VI Membranes of liver mitochondria. Arch. Biochem. Biophys., 125:9811012.

Ardail, D., Lermé, F., and Louisot, P. (1990a) Further characterization of mitochondrial contact sites: Effect of short chain alcohols on membrane fluidity and activity. Biochem. Biophys. Res. Commun., 173:878-885.

Ardail, D., Lerme, F., and Loisot, P. (1991) Involvement of contact sites in phosphatidylserine import into liver mitochondria. J. Biol Chem., 266:7978-7981.

Ardail, D., Privat, J.P., Charlier, M., Levrat, C., Lerme, F., and Louisot, P. (1990b) Mitochondrial contact sites: Lipid composition and dynamics. J. Biol. Chem., 265:18797-19902.

Attardi, G., and Schatz, G. (1988) Biogenesis of mitochondria. Annu. Rev. Cell Biol. 4:289-333.

Baker, K.P., and Schatz, G. (1991) Mitochondrial proteins essential for viability mediate protein import into yeast mitochondria. $\mathrm{Na}$ ture, 349:205-208.

Biermans, W., Bakker, A., and Jacob, W. (1990) Contact sites between inner and outer mitochondrial membranes: A dynamic microcompartment for creatine kinase activity. Biochim. Biophys. Acta, 1018:225-228.

Biermans, W., Bernaert, I., de Bie, M., Nijs, B., and Jacob, W. (1989) Ultrastructural localization of creatine kinase activity in the con- 
tact sites between inner and outer mitochondrial membranes of rat myocardium. Biochim. Biophys. Acta, 974:74-80.

Brdiczka, D. (1991) Contact sites between mitochondrial envelope membranes. Structure and function in energy- and protein-transfer. Biochim. Biophys. Acta, 1071:291-312.

Brdiczka, D., Bücheler, K., Kottke, M., Adams, V., and Kumar Nalam, V. (1990) Characterization and metabolic function of mitochondrial contact sites. Biochim. Biophys. Acta, 1018:234-238.

Eilers, M., and Schatz, G. (1986) Binding of a specific ligand inhibits import of a purified precursor protein into mitochondria. Nature, $322: 228-232$.

Glick, B., Wachter, C., and Sehatz, G. (1991) Protein import into mi tochondria: Two systems acting in tandem? Trends Cell Biol., 1:99103 .

Hackenbrock, C.R. (1966) Ultrastructural bases for metabolically linked mechanical activity in mitochondria. J. Cell Biol., 30:269297.

Hackenbrock, C.R. (1968) Chemical and physical fixation of isolated mitochondria in low-energy and high energy states. Proc. Natl. Acad. Sci. U.S.A., 61:598-605.

Hackenbrock, C.R., and Miller, K.J. (1975) The distribution of anionic sites on the surface of mitochondrial membranes. J. Cell Biol., 65: $615-630$

Hartl, F.-U., and Neupert, W. (1990) Protein sorting to mitochondria: Evolutionary conservations of folding and assembly. Science 247 $930-938$.

Hartl, F.-U., Pfanner, N., Nicholson, D.W., and Neupert, W. (1989) Mitochondrial protein import. Biochim. Biophys. Acta, 988:1-45.

Hines, V., Brandt, A., Griffiths, G., Horstmann, H., Brütsch, H., and Schatz, G. (1990) Protein import into yeast mitochondria is accelerated by the outer membrane protein MAS70. EMBO J., 9:31913200 .

Hwang, S., Jascur, T., Vestweber, D., Pon, L., and Schatz, G. (1989) Disrupted yeast mitochondria can import precursor proteins directly through their inner membrane. J. Cell Biol. 109:487-493.

Kellems, R.E., Allison, V.F., and Butow, R.A. (1975) Cytoplasmic type $80 \mathrm{~S}$ ribosomes associated with yeast mitochondria. J. Cell Biol. 65:1-14.

Kiebler, M., Pfaller, R., Söllner, T., Griffiths, G., Horstmann, H. Pfanner, N., and Neupert, W. (1990) Identification of a mitochondrial receptor complex required for recognition and membrane insertion of precursor proteins. Nature, 348:610-616.

Knoll, G., and Brdiczka, D. (1983) Changes in freeze-fractured mitochondrial membranes correlated to their energetic state. Dynamic interactions of the boundary membranes. Biochim. Biophys. Acta 733:102-110.

Lill, R., Stuart, R.A., Drygas, M.E., Nargang, F.E., and Neupert, W (1992) Import of cytochrome $\mathrm{c}$ heme lyase into mitochondria: A novel pathway to the intermembrane space. FMBO J., 11:449-456.

Lithgow, T., Timms, M., Hoj, P.B., Hoogenraad, N.J. (1991) Identification of a GTP-binding protein in the contact sites between inner and outer mitochondrial membranes. Biochem. Biophys. Res. Commun., 180:1453-1459.

Malhotra, S.K., and Tewari, J.P. (1991) Contact sites between mitochondrial membranes: Comments. Cytobios, 68:91-94

Martin, J., Mahlke, K., and Pfanner, N. (1991) Role of an energized inner membrane in mitochondrial protein import. J. Biol. Chem., 266:18051-18057.

Marzuki, S., and Hibbs, A.R. (1986) Are all mitochondrial translation products synthesized on membrane-bound ribosomes? Biochim. Biophys. Acta, 866:120-124.

McEnery, M.W., Snowman, A., Trifiletti, R.R., and Snyder, S. (1992) Isolation of the mitochondrial benzodiazepine receptor: Association with the voltage dependent anion channel and the adenine nucleotide carrier. Proc. Natl. Acad. Sci. U.S.A., 89:3170-3174.

Murakami, H., Blobel, G., and Pain, D. (1990) Isolation and characterization of the gene for a yeast mitochondrial import receptor. Nature, 347:488-491.

Nicolay, K., Rojo, M., Wallimann, T., Demel, R., and Hovius, R. (1990) The role of contact sites between inner and outer mitochondria membrane in energy transfer. Biochim. Biophys. Acta, 1018:229233.

Ohlendieck, K., Riesinger, I., Adams, V., Krause, J., and Brdiczka, D. (1986) Enrichment and biochemical characterization of boundary membrane contact sites from rat-liver mitochondria. Biochim. Biophys. Acta, 860:672-689.
Pain, D., Murakami, H., and Blobel, G. (1990) Identification of a receptor for protein import into mitochondria. Nature, 347:444-449. Pfanner, N, , and Neupert, W. (1990) The mitochondrial protein import apparatus. Annu. Rev. Biochem., 59:331-353.

Pfanner, N., Rassow, J., van der Klei, I.J., and Neupert, W. (1992) A dynamic model of the mitochondrial import machinery. Cell, 68: 999-1002.

Pfanner, N., Söllner, T., and Neupert, W. (1991) Mitochondrial import receptors for precursor proteins. Trends Biochem. Sci., 16:63-67.

Pon, L., Moll, T., Vestweber, D., Marshallsay, B., and Schatz, G. (1989) Protein import into mitochondria: ATP-dependent protein translocation activity in a submitochondrial fraction enriched in membrane contact sites and specific proteins. J. Cell Biol., 109: 2603-2616.

Rassow, J., Guiard, B., Wienhues, U., Herzog, V., Hartl, F.-U., and Neupert, W. (1989) Translocation arrest by reversible folding of a precursor protein imported into mitochondria. A means to quantitate translocation contact sites. J. Cell Biol., 109:1421-1428.

Rassow, J., Hartl, F.U., Guiard, B., Pfanner, N., and Neupert, W. (1990) Polypeptides traverse the mitochondrial envelope in an extended state. FEBS Lett., 275:190-194.

Rassow, J., and Pfanner, N. (1991) Mitochondrial preproteins en route from the outer membrane to the inner membrane are exposed to the intermembrane space. FEBS Lett., 293:85-88.

Rojo, M., Hovius, R., Demel, R.A., Nicolay, K., and Walliman, T. (1991) Mitochondrial creatine kinase mediates contact formation between mitochodnrial membranes. J. Biol. Chem., 266:2029020295.

Schleyer, M., and Neupert, W. (1985) Transport of proteins into mitochondria: Translocation intermediates spanning contact sites between outer and inner membranes. Cell, 43:339-350.

Schwaiger, M., Herzog, V., and Neupert, W. (1987) Characterization of translocation contact sites involved in the import of mitochondrial proteins. J. Cell Biol., 105:235-246.

Söllner, T., Griffiths, G., Pfaller, R., Pfanner, N., and Neupert, W. (1989) MOM19, an import receptor for mitochondrial precursor proteins. Cell, 59:1061-1070.

Söllner, T., Pfaller, R., Griffiths, G., Pfanner, N., and Neupert, W. (1990) A mitochondrial import receptor for the ADP/ATP carrier. Cell, 62:107-115

Söllner, T., Rassow, J., Wiedmann, M., Schlossmann, J., Keil, P., Neupert, W., and Pfanner, N. (1992) Mapping of the protein import machinery in the mitochondrial outer membrane by cross-linking of translocation intermediates. Nature, 355:84-87.

Stuart, R.A., Nicholson, D.W., and Neupert, W. (1990) Early steps in mitochondrial protein import: Receptor functions can be substituted by the membrane insertion activity of apocytochrome c. Cell, 60: $31-34$.

Suissa, M., and Schatz, G. (1982) Import of proteins into mitochondria. Translatable mRNA's for imported mitochondrial proteins are present in free as well as mitochondria-bound cytoplasmic polysomes. J. Biol. Chem., 257:13048-13055.

Venetië, R., van, and Verkleij, A.J. (1982) Possible role of non-bilayer lipids in the structure of mitochondria: A freeze-fracture electron microscopy study. Biochim. Biophys. Acta, 692:397-405.

Vestweber, D., Brunner, J., Baker, A., and Schatz, G. (1989) A 42K outer-membrane protein is a component of the yeast mitochondrial protein import site. Nature, 341:205-209.

Vestweber, D, and Schatz, G. (1988) A chimeric mitochondrial precursor protein with internal disulfide bridges blocks import of authentic precursors into mitochondria and allows quantitation of import sites. J. Cell Biol., 107:2037-2043.

Vignais, P.V., Huet, J., and André, J. (1969) Isolation and characterization of ribosomes from yeast mitochondria. FEBS Lett., 3:177181.

Watson, K. (1972) The organization of ribosomal granules within mitochondrial structures of aerobic and anaerobic cells of Saccharomyces cerevisiae. J. Cell Biol., 55:721-726.

Werner, S., and Neupert, W. (1972) Functional and biogenetical heterogeneity of the inner membrane of rat-liver mitochondria. Eur. J. Biochem., 25:379-396.

Wienhues, U., and Neupert, W. (1992) Protein translocation across mitochondrial membranes. Bioessays, 14:17-23.

Zara, V., Rassow, J., Wachter, E., Tropschug, M., Palmieri, F., Neupert, W., and Pfanner, N. (1991) Biogenesis of the mitochondrial phosphate carrier. Eur. J. Biochem., 198:405-410 